\title{
Behandlungsbewertungen von Jugendlichen im Verlauf einer stationär-psychiatrischen Behandlung
}

[Consumer satisfaction trajectories of adolescents during inpatient psychiatric treatment]

\author{
Ferdinand Keller ${ }^{1}$, Susanne Peter ${ }^{2}$, Jörg M. Fegert ${ }^{1}$, Alexander \\ Naumann ${ }^{3}$, Lutz Goldbeck ${ }^{1}$
}

1) Klinik für Kinder- und Jugendpsychiatrie/Psychotherapie, Universitätsklinikum Ulm

2) Zentrum für Psychiatrie „Die Weissenau“

3) Klinik für Kinder- und Jugendpsychiatrie und Psychotherapie, Niedersächsisches Landeskrankenhaus Lüneburg 
Behandlungsbewertungen von Jugendlichen im Verlauf einer stationärpsychiatrischen Behandlung

Fragestellung: Einschätzungen zur Prozessqualität

psychiatrisch/psychotherapeutischer Behandlung erfolgen meist rückblickend bei Entlassung, weshalb bisher wenig bekannt ist über Veränderungen in der

Zufriedenheit mit den Therapien und bei verwandten Aspekten wie Partizipation und Motivation. Es wird untersucht, ob computergestützte Befragungen im stationären Verlauf machbar sind, die Fragen differenzieren und ob sich Gruppenunterschiede im Verlauf und in der Variabilität ergeben. Methodik: 88 Jugendliche (48 Mädchen, 44 Jungen) im Alter von 13 bis 18 Jahren, die sich auf jugendpsychiatrischen Abteilungen zweier Kliniken in Behandlung befanden, wurden zwei Mal wöchentlich mit 16 Items zu den genannten Themen befragt; insgesamt stehen 1003 Befragungen zur Verfügung, der Median der Befragungen pro Person liegt bei 8. Ergebnisse: Psychometrische Analysen sprechen für eine gute Differenziertheit der Fragen. Auswertungen mit einem Wachstumskurvenmodell, das wegen der unterschiedlich langen Zeitreihen und vereinzelt auftretenden fehlenden Werten bevorzugt wurde, zeigen, dass in den personenspezifischen Verlaufskurven die Niveaus sehr unterschiedlich sind. In der globalen Zufriedenheit bleiben sie im Verlauf der Behandlung relativ stabil. Im Bereich von Mitbestimmung ergeben sich unterschiedliche Trends und auch ein Unterschied zwischen den beiden beteiligten Kliniken, der auf die unterschiedliche Teamerfahrung zurückzuführen sein dürfte, während Alter, Geschlecht und Diagnose nur eine geringe Rolle spielen. Schlussfolgerung: Eine begleitende computergestützte Datenerhebung unter stationären Behandlungsbedingungen zur Evaluation der Prozessqualität bei Jugendlichen hat sich als praktikabel erwiesen und ergänzt die ergebnisorientierte Forschung um eine prozessorientierte Perspektive.

Schlüsselworte: Behandlungszufriedenheit; Partizipation; Jugendpsychiatrie; Wachstumskurvenanalyse; Prozessforschung. 
Consumer satisfaction trajectories of adolescents during in-patient psychiatric treatment

Objective: Patient satisfaction with in-patient treatment and other aspects of process quality is usually examined cross-sectionally (e.g. at discharge).

Therefore, the knowledge about change of patient satisfaction and related issues such as participation and motivation during the treatment period is limited. This study describes the development of a questionnaire concerning these topics and the course and variability in these domains for different subgroups. Method: The study group comprises 88 adolescents (48 female, 40 male; age between 13 and 18 years) who delivered altogether 1003 data points (median per person is 8). The questionnaire consisted of 16 items that were administered by computer twice a week at adolescent wards in two psychiatric departments. Statistical analysis was done using hierarchical linear modeling (growth curve models) that is well suited for the analysis of data with different number of assessment points and missing data. Results: Psychometric properties of the questions were good. The trajectories in all items are very heterogeneous regarding the level. The trends (slope of the growth curves) are around 0 in general satisfaction, i.e. the adolescents stay on their different levels during the treatment period. Concerning participation, however, there are diverging trends, in particular between the two departments which may reflect the difference in treatment experience of the teams, whereas age, gender and diagnosis (internalizing vs. externalizing) play a minor role. Conclusion: Evaluation of process characteristics has turned out to be feasible in clinical routine and promising for further insights into process quality.

Key words: adolescent psychiatry; growth curve analysis; patient satisfaction; process quality 


\section{Einleitung}

Die Zufriedenheit psychiatrischer Patienten mit ihrem stationären Aufenthalt gilt inzwischen als wichtiges Qualitätskriterium einer Einrichtung, obwohl die Erfassung dieses Konstrukts immer wieder kritisiert und hinterfragt wird, sowohl hinsichtlich seiner theoretischen Konzeptualisierung als auch bezüglich methodischer Unzulänglichkeiten und Probleme (Gruyters \& Priebe, 1994; Leimkühler \& Müller, 1996; Teague, 2000).

Bemängelt wird vor allem eine undifferenzierte Abfrage globaler Zufriedenheit (die dann meist sehr hoch ausfällt), die Gleichsetzung der Patientenzufriedenheit mit dem betriebswirtschaftlichen Konzept der Kundenorientierung (Dür, Grossmann \& Schmied, 2000) und die geringe Differenziertheit des Konstrukts, da sich bei einer psychometrischen Analyse gängiger Erhebungsinstrumente zumeist eindimensionale Lösungen ergeben. Trotz dieser Einschränkungen empfiehlt Richter (2004) die Erhebung der Patientenzufriedenheit als Indikator für die Qualität psychiatrischer Behandlung. Auch für die Evaluation kinder- und jugendpsychotherapeutischer Behandlungen regen Weisz und Kazdin (2003) die Integration der „Konsumentenperspektive“ an. Vor dem Hintergrund der bisher als unzulänglich bewerteten Implementierung evidenzbasierter Psychotherapiemethoden in die klinische Praxis (Weisz \& Jensen, 2001) könnte das Konzept der Behandlungszufriedenheit als Indikator des therapeutischen Bündnisses zwischen Behandlern und Patient ein wichtiger unspezifischer Wirkfaktor in der Psychotherapie sein (Chatoor \& Krupnick, 2001).

In der Kinder- und Jugendpsychiatrie liegen einige neuere Untersuchungen vor, in denen empirische Befunde zur Erhebung und Bewertung der Behandlungszufriedenheit vorgestellt werden. Mattejat und Remschmidt (1998a) entwickelten mit dem FBB einen Fragebogen, in dem sie hauptsächlich den Komplex „Akzeptabilität der Behandlung“ erheben, d.h. die Beziehung zu Therapeuten und zu den Mitpatienten sowie die Mitarbeit 
bei therapeutischen Maßnahmen. Ein explizit auf die therapeutische Beziehung zugeschnittener Fragebogen wurde auch von Kronmüller et al. (2003) vorgelegt. Studien mit Schwerpunkt auf einer weiter gefassten Behandlungszufriedenheit im stationären Bereich (z.T. auch mit Fragen zur Strukturqualität) liegen ebenfalls vor (Bredel et al., 2004; Distler, 2002; Keller et al., 2004; Konopka, 2003).

Obwohl damit eine Reihe von Erfassungsinstrumenten zur Verfügung steht, bleiben Fragen offen. Unter die noch wenig bearbeiteten Forschungsfragen zählen MöllerLeimkühler \& Dunkel (2003) die Frage, ob sich Patientenurteile im Behandlungsverlauf wesentlich ändern, und sie vermuten, dass die zeitliche Stabilität von Behandlungszufriedenheit hoch ist. Mit Querschnittserhebungen, wie sie bislang meist vorgenommen wurden, lässt sich diese Frage allerdings nicht beantworten, und zusätzlich entsteht dabei das Problem, dass wichtige Aspekte der Prozessqualität nur summarisch im Rückblick erhoben werden können. Auch Richter (2004) hält die Ergänzung durch Längsschnittdaten oder Zeitreihen für methodisch geboten, da Querschnittsdaten zufälligen Einflüssen unterliegen können, die sich nicht ohne weiteres erschließen lassen.

Freudenmann und Spitzer (2001) berichten über Ergebnisse einer täglich durchgeführten Einschätzung der Ergebnisqualität in einer Erwachsenenpsychiatrie, die mittels eines computergestützten Befragungssystems vorgenommen wurde. Im Itempool sind auch Fragen zur Zufriedenheit enthalten, doch werden eigentliche Prozessaspekte nur anhand zweier Einzelfalldarstellungen kurz angesprochen. In der Kinder- und Jugendpsychiatrie wurde u.W. bisher noch keine Studie zu Verlaufsaspekten von Zufriedenheit und Mitbestimmung publiziert, abgesehen von einer eigenen Pilotstudie zum Verlauf der Behandlungszufriedenheit (Keller et al., 2003). Dabei zeigte sich, dass die Ausgangswerte der allgemeinen Zufriedenheit sowie das Zurechtkommen auf der Station wie zu erwarten sehr unterschiedlich ausgeprägt war, dass aber die Jugendlichen während des stationären Aufenthaltes im Wesentlichen auf ihrem Niveau verblieben und damit z.B. 
keine Zunahme der Zufriedenheit nachweisbar war. Methodisch wurde dazu eine Auswertung mittels Wachstumskurvenanalysen angewendet, bei denen die Zeit als quantitative Variable einbezogen wird, so dass die Daten bei solchen naturalistischen Designs mit ungleich vielen Messzeitpunkten und zwischenzeitlich immer wieder fehlenden Werten bei der Schätzung von personenspezifischen Verläufen vollständig berücksichtigt werden können. Gruppenunterschiede konnten aber aufgrund der dafür zu kleinen Stichprobe nicht adäquat überprüft werden.

Die vorliegende Studie beschäftigt sich daher aus inhaltlichen und methodischen Gründen mit der Replikation dieses Ergebnisses an einer größeren Stichprobe unter Einbeziehung zusätzlicher Fragen, die sich auf Mitbestimmung, Autonomie, Information und Motivation beziehen. Diese bisher vernachlässigten Dimensionen wurden aufgenommen bzw. erweitert, nachdem Wiethoff et al. (2003) fanden, dass erlebte Partizipation einen signifikanten Beitrag zum Therapieerfolg leistete. Im Einzelnen sollte dabei untersucht werden:

1) Die Geeignetheit der Items für Verlaufserhebungen, indem eine psychometrische Analyse der Items, eine Hauptkomponentenanalyse zur Abschätzung ihrer Dimensionalität und eine externe Validierung mit dem Inventar zur Lebensqualität von Kindern und Jugendlichen ILK (Mattejat et al., 1998) vorgenommen werden.

2) Eine Überprüfung der personenspezifischen Heterogenität der Verlaufskurven (= Replikation des Ergebnisses “verschiedene Niveaus, aber wenig Änderung” aus der Pilotstudie) und die zu erwartenden Einflüsse von Alter, Geschlecht und Diagnose. 3) Eine besondere Rolle spielt der Vergleich des Behandlungserlebens zwischen den beiden beteiligten Kliniken (Weissenau und Ulm), die unter anderem auch den Unterschied zwischen einem etablierten und einem sich ganz neu orientierenden Team abbilden.

4) Die intraindividuelle Variabilität bei Personen aus unterschiedlichen diagnostischen Subgruppen, insbesondere Persönlichkeitsstörungen des emotional instabilen Typs. 


\section{Methodik}

\subsection{Stichprobe}

Der Erhebungszeitraum lag zwischen Frühjahr und Herbst 2002; während dieser Zeit wurden konsekutiv alle neu aufgenommenen Patienten der beiden Jugendlichen-Stationen am Zentrum für Psychiatrie Weissenau, einer regelversorgenden Klinik, und der Jugendstation der Universitätsklinik für Kinder- und Jugendpsychiatrie/Psychotherapie Ulm untersucht. Voraussetzung für die Teilnahme war das Einverständnis der Jugendlichen, ein hinreichendes Verständnis und Lesefähigkeit sowie eine ausreichende Kommunikationsfähigkeit. Die Datenerhebung fand zweimal in der Woche dienstags und freitags jeweils am Nachmittag statt. Weniger als 5 \% aller im Erhebungszeitraum behandelten Patienten konnten nicht in die Untersuchung einbezogen werden, weil sie der Befragung nicht zustimmten oder aufgrund schwerster psychopathologischer Auffälligkeiten nicht teilnehmen konnten. Alle Teilnehmer begannen innerhalb der ersten Tage nach Aufnahme und beantworteten die Fragen bis zur Entlassung.

Die Stichprobe umfasst somit 88 Patienten mit insgesamt 1003 Befragungszeitpunkten. Im Mittel liegen 11,4 Zeitpunkte pro Person vor, doch verweist der Median von 8 auf den Einfluss einiger Patienten, von denen viele Zeitpunkte vorliegen. Der Großteil liegt zwischen 4 und 14,5 (25\% und 75\% Quartile). Neun Patienten weisen mehr als 25 Zeitpunkte auf, mit einem Maximum von 49. Bedingt durch klinikspezifische Besonderheiten unterscheidet sich die durchschnittliche Aufenthaltsdauer in Weissenau und Ulm, was sich auch in der Zahl der erfassten Untersuchungszeitpunkte zeigt. Die 52 Patienten aus Weissenau weisen insgesamt 434 Zeitpunkte auf, mit einem Median von 6,5 und Quartilen von 3 und 10 (25\% und 75\%). In Ulm werden von 36 Patienten insgesamt 569 Zeitpunkte erfasst, mit einem Median bei 14 und Quartilen von 8 und 22.

Bei den 88 Studienpatienten handelte es sich um 48 Mädchen und 40 Jungen; von 
den Mädchen liegen 551 Zeitpunkte vor und von den Jungen sind es 452, so dass die Mädchen vergleichsweise mit etwas mehr Zeitpunkten vertreten sind. Die Jugendlichen waren zwischen 14 und 18,4 Jahre alt, das mittlere Alter betrug 16,0 Jahre (SD=1,0), der Median liegt bei 16,1. Die Klinikunterschiede sind mit mittleren 16,1 bzw. 16,0 Jahren minimal. Die diagnostische Verteilung gemäß den Hauptdiagnosen ist folgendermaßen: In die Gruppe der Schizophrenie (ICD-10 F2) fielen neun Personen, sieben Patienten waren depressiv (F3). Unter “Neurosen” und emotionale Störungen (F4, F93) wurden 21 Patienten zusammengefasst. Als Essstörungen (F50) wurden neun Patienten diagnostiziert, und die Hauptdiagnose Persönlichkeitsstörung (F6) wiesen 10 Patienten auf. Die größte Gruppe bestand aus externalisierenden Störungen wie Störungen des Sozialverhaltens, ADHS usw. (F90, F91, F92) mit 26 Personen. Alle übrigen Personen wurden den internalisierenden Störungen zugeordnet, auch eine Restkategorie, in die noch sechs Personen fielen.

\subsection{Erhebungsinstrumente und Ablauf}

Die Erhebung erfolgte computergestützt durch zwei externe Untersucher auf der jeweiligen Station in einem separaten Raum, wo jeweils nur ein Jugendlicher die Fragen beantwortete. Die Fragen wurden einzeln auf dem Bildschirm vorgegeben, darunter befand sich zur Beantwortung eine fünfstufige Antwortskala mit den beiden Extremen „stimmt überhaupt nicht“ und „stimmt vollkommen“; mit der Maus konnte dann das gewählte Kästchen angekreuzt werden. Danach erschien die nächste Frage. Bei einigen Fragen wurde zusätzlich die Antwortkategorie „trifft nicht zu“ bzw. „heute nicht stattgefunden“ mit aufgeführt.

\subsection{Statistische Auswertung}

Neben Standardmethoden werden zur Auswertung hierarchische lineare Modelle, speziell 
ein Wachstumskurvenmodell, das meist als random regression-Modell (RRM) bezeichnet wird, herangezogen. Damit ist es möglich, personenspezifische Verläufe (Trajektorien) zu schätzen, in deren Schätzprozess sowohl die individuellen Verlaufsdaten als auch die Verlaufskurve der Gesamt- bzw. Subgruppe (z.B. diagnostische Subgruppe), der die Person angehört, eingehen (Keller, 2003; Raudenbush, 2001; Singer, 2002). Die Berechnungen zum RRM wurden mit der Prozedur MIXED (Littell et al., 1996) aus dem SAS in der Version 8.2 vorgenommen. Die übrigen Analysen erfolgten ebenfalls mit den entsprechenden Prozeduren aus dem SAS. Im RRM werden zwar nur zwei Parameter (Achsenabschnitt und Steigung) für jede Person geschätzt, doch bekommen die lang andauernden Behandlungsverläufe durch die vielen Zeitpunkte ein wesentlich höheres Gewicht; gleichzeitig ist die Annahme eines linearen Verlaufes über die ganze Zeit nicht unbedingt gerechtfertigt, weshalb für die folgenden Auswertungen durchgängig die ersten beiden Monate (60 Tage) gewählt wurden; darin sind einerseits knapp 80\% der Daten enthalten, andererseits aber die Personen mit den langen Verläufen nicht zu stark gewichtet. Die Berechnungen basieren somit auf $t=783$ bzw. bei den Fragen nach Therapien auf $\mathrm{t}=360$ und bei denjenigen nach Medikamenten auf $\mathrm{t}=391$.

Die Variabilität in den Verläufen lässt sich über die Varianz der Einzelwerte über die Zeit hinweg operationalisieren. Allerdings entsteht dabei das Problem, dass Varianz und Trends konfundiert sind und die Varianz daher allein durch einen Trend im Verlauf vergrößert wird. Um eine trendbereinigte Varianz zu erhalten, wurden daher die personenspezifischen Regressionsgeraden aus dem RRM herangezogen. Die Abweichungen (Residuen) von dieser jeweiligen Geraden wurden bestimmt und ihre Varianz berechnet. Für jede Person steht somit pro Frage ein neuer Messwert für die zeitliche Variabilität zur Verfügung, der dann in die Gruppenvergleiche mittels nonparametrischer Rangvarianzanalyse nach Kruskal-Wallis eingeht. 


\section{Ergebnisse}

\subsection{Ergebnisse zum Messinstrument}

\subsubsection{Häufigkeitsauszählungen zu den Einzelfragen}

Die Gesamtverteilung der Angaben in den einzelnen Antwortkategorien jeder Frage ist aus Platzgründen nicht aufgeführt, zeigt aber, dass in allen Fragen die zur Verfügung stehenden fünf Kategorien auch benutzt wurden. Insgesamt ist eine Verschiebung nach rechts zu verzeichnen und die Mittelwerte (Tab. 1) liegen alle über 3 (mit Ausnahme von Frage 13, bei der niedrige Werte vorteilhaft sind) und damit im positiven Bereich. Den höchsten Wert erreicht Frage 6 („,mit anderen Jugendlichen verstanden“), und als nächstes folgt bereits die Erfahrung, von den Therapeuten ernst genommen zu werden (Frage 8). Auch ihre Motivation zur weiteren Behandlung (Frage 10) und zur Mitarbeit bei den Therapien (Frage 11) schätzen die Jugendlichen als eher hoch ein. Die allgemeine Zufriedenheit (Frage 2) ist dagegen verhältnismäßig niedrig. Zusammenfassend kann daher angenommen werden, dass die Fragen des Prozessfragebogens differenzieren und auch keine massiven Boden- und Deckeneffekte auftreten. hier etwa Tab. 1

\subsubsection{Zusammenhänge mit Alter}

Alterseffekte sind in der Zufriedenheit und insbesondere in der Mitbestimmung zu erwarten, weshalb sie hier separat und vorab betrachtet werden. Die Einschätzung der Zusammenhänge erfolgt dabei rein deskriptiv, da eine Signifikanzbestimmung der Korrelationskoeffizienten wegen der Abhängigkeit der Messungen problematisch ist. Die höchste Korrelation besteht mit „über Behandlung informiert“ (Frage 9) mit r=-0,23, d.h. ältere Jugendliche fühlen sich eher weniger über ihre Behandlung informiert; ebenso haben sie auch weniger „Lust, mitzuarbeiten“ ( $\mathrm{r}=-0,18)$. Medikamente werden als weniger 
hilfreich eingeschätzt ( $\mathrm{r}=-0,25)$, gleichzeitig erleben ältere Jugendliche aber kaum mehr Nebenwirkungen $(\mathrm{r}=0,12)$.

\subsubsection{Dimensionalität der Fragen}

Für die Daten des jeweils dritten Messzeitpunkts (analog Freudenmann \& Spitzer, 2001) ergeben sich in einer Hauptkomponentenanalyse drei Komponenten (Eigenwerte 5,51; 1,95 und 1,28), die insgesamt 62,8\% der Varianz aufklären (nach varimax-Rotation etwa gleich große Varianzanteile davon) und mit „Behandlungsmotivation”, „Zufriedenheit“ und “Mitbestimmung/ernst genommen werden” bezeichnet werden können (Tab. 1). Die Fragen zur Mitbestimmung bilden damit wie erwartet einen eigenständigen Faktor, wenn auch „über Behandlung informiert“ hier (noch) nicht ausreichend lädt; bei Heranziehung aller Messzeitpunkte (was wegen der teilweise abhängigen Messungen nur mit Vorsicht interpretiert werden kann) wechselt diese Frage zum Mitbestimmungsfaktor.

\subsubsection{Externe Validierung am ILK}

Die Korrelationsanalyse zwischen den Zufriedenheitsfragen und der einmal wöchentlich zeitgleich vorgenommenen Selbsteinschätzung der Jugendlichen im Inventar zur Lebensqualität (ILK) ergibt Zusammenhänge in der erwarteten Richtung (vgl. Tab. 2). Deutliche Korrelationen zeigen sich mit der Gesamtbeurteilung der Lebensqualität und mit der subjektiven psychischen Gesundheit. Einige moderate Zusammenhänge sind auch mit den ILK-Skalen Problembelastung und Behandlungsbelastung zu verzeichnen. Auch die Einschätzung, man sei seinem Problem ausgeliefert, zeigt wie erwartet eine deutliche positive Korrelation mit dem psychischen Befinden und der allgemeinen Lebensqualität. Bei der Interpretation der Korrelationskoeffizienten ist die unterschiedliche Polung der Antwortskalen und das unterschiedliche Zeitfenster der beiden Instrumente (ILK: zurückliegende Woche; Zufriedenheit: heutiger Tag) zu berücksichtigen. 
hier etwa Tab. 2

\subsection{Verlauf und personenspezifische Heterogenität in Niveau und Trend}

\subsubsection{Allgemeine Verlaufseffekte}

Die Betrachtung gemittelter Verläufe in den einzelnen Fragen ist dadurch eingeschränkt, dass die Mittelwerte auf wechselnden Stichprobengrößen beruhen, da die Zahl der Jugendlichen mit zunehmender Anzahl der Zeitpunkte abnimmt. Zur Berechnung genereller Trends über die Zeit wird daher der Haupteffekt „Zeit“ im Rahmen des RRM herangezogen. Tab. 3 enthält die entsprechenden $p$-Werte, und es zeigt sich, dass nur wenige Auswertungen zum reinen Verlaufseffekt signifikant sind. Einen deutlich signifikanten Zeiteffekt weist nur das Auskommen mit Betreuern (Frage 5) auf, in dem sich insgesamt eine Abnahme in der (anfangs sehr hohen) Zufriedenheit mit den Betreuern manifestiert, die sich auch in der Einschätzung, ob man von den Betreuern ernst genommen (Frage 7) und in der Frage der Mitbestimmung (Frage 3) tendenziell signifikant widerspiegelt. Außerdem ergibt sich eine Verbesserung bei „Medikamente helfen“, die aber nach Inspektion der Verlaufskurven auf einige wenige sehr deutliche Verbesserungen zurückzuführen ist, während die meisten geschätzten Verlaufskurven allenfalls minimal zu- bzw. abnehmen. Bei allen übrigen Fragen kann daher für die Gesamtgruppe keine gleichgerichtete mittlere Zu- oder Abnahme angenommen werden. hier etwa Tab. 3

\subsubsection{Heterogenität der Trajektorien}

Eine zweite Analyse bezieht sich auf die Heterogenität der Achsenabschnitte und der Steigungen (die beiden random effects im RRM, deren Signifikanz über eine z-Statistik geprüft werden kann (vgl. Singer, 2002, aber auch die kritische Diskussion in Verbeke \& Molenberghs, 2000, S. 64f., die sich insbesondere auf Signifikanztests bei den 
Achsenabschnitten bezieht)). Bei der Unterschiedlichkeit der Ausgangswerte in den personenspezifischen Verlaufsgeraden ergeben sich über alle Fragen hinweg sehr signifikante Effekte, d.h. die Heterogenität der Ausgangswerte der Regressionsgeraden ist stark ausgeprägt (nicht eigens in Tab. 3 dargestellt wegen der erwähnten Testproblematik und der gleichzeitig geringen inhaltlichen Bedeutung). Bei den Steigungskoeffizienten, die die Unterschiedlichkeit in der Richtung der Veränderung anzeigen, ergeben sich einige signifikant heterogene Trends (Tab. 3). Besonders tritt dies bei der Frage "sinnvoll, dass weiter hier” auf, was auf eine sehr unterschiedliche Entwicklung während des Aufenthaltes hindeutet. Signifikante Effekte finden sich auch bei den Fragen im Bereich Mitbestimmung. Beim “Essen geschmeckt” ergibt sich ebenfalls eine deutlich signifikante Aufspaltung in zunehmend bessere und schlechtere Bewertung des Essens. Auch die Hilfe durch Medikamente wird über die Zeit unterschiedlich erlebt (vgl. oben). Auf einem weitgehend stabilen Niveau verbleiben dagegen die allgemeine Zufriedenheit, das Auskommen mit den anderen Jugendlichen und auch „Lust mitzuarbeiten“.

\subsection{Gruppenspezifische Unterschiede}

\subsubsection{Geschlecht}

Die Auswertungen zum Geschlechtseffekt ergaben keine auf dem 5\%-Niveau signifikanten Gruppenunterschiede. Tendenziell $(\mathrm{p}<.10)$ sind Mädchen weniger zufrieden (Frage 2) und haben weniger “Lust, mitzuarbeiten”, meinen aber, Medikamente helfen besser. Signifikante Interaktionseffekte traten nicht auf.

\subsubsection{Internalisierende vs. externalisierende Störungen}

Die Gruppe der Jugendlichen mit externalisierenden ist im Vergleich zu der mit internalisierenden Störungen insgesamt zufriedener mit ihren Therapien, kommt besser mit den Betreuern aus und versteht sich auch besser mit den anderen Jugendlichen (vgl. Tab. 3, 
Haupteffekte). Dabei sind sie zudem auch motivierter (Fragen 10 und 11). Beim Eindruck, man sei seinen Problemen ausgeliefert, ergibt sich eine tendenziell signifikant erhöhte Zustimmungsrate bei den Jugendlichen mit internalisierenden Störungen.

\subsubsection{Klinikunterschiede}

Im Vergleich der beiden beteiligten Kliniken fällt auf, dass sich keine signifikanten Differenzen ergeben, wenn man nur die generellen Unterschiede (Haupteffekt Klinik) betrachtet (Tab. 3). Bei der Einbeziehung des Zeitfaktors (Interaktion Klinik x Zeit) ergeben sich allerdings einige interessante Verlaufsunterschiede, die sich v.a. im Bereich Mitbestimmung manifestieren. Insbesondere zeigen die Verlaufskurven für Frage 3, dass die Jugendlichen in Ulm zwar ebenfalls in großem Maße mitbestimmen können, doch gelingt es in Weissenau, das Gefühl der Mitbestimmung über die Zeit hinweg noch zu steigern. Die anderen Fragen aus diesem Bereich weisen ähnliche Tendenzen auf und die Summe dieser Fragen $(3,5,7,9,16)$ ergibt ein analog signifikantes Ergebnis $(F(1,693)=$ 7,47, p=0,006). Eine kombinierte Varianzanalyse mit den beiden Faktoren Klinik und Diagnose ergibt eine signifikante Dreifachinteraktion Klinik x Diagnose x Zeit $(\mathrm{F}(1,691)=6,69, \mathrm{p}=0,010)$ für den Summenwert Mitbestimmung, der vor allem aus der zunehmend unterschiedlichen Mitsprachebeurteilung der Jugendlichen mit internalisierenden Störungen resultiert.

\subsection{Variabilität}

Erhöhte intraindividuelle Schwankungen wurden v.a. für die Gruppe der emotional instabilen Persönlichkeitsstörungen vermutet, was sich aber in den Daten nicht nachweisen ließ. Bei einem Vergleich der inhaltlich interessantesten und ausreichend besetzten diagnostischen Subgruppen (F4, F5, F6, F9) zeigte sich, dass nicht die 
zumeist eine höhere Variabilität aufwies. Für den Summenwert Mitbestimmung liegt sie mit SD=3,4 signifikant über den Standardabweichungen der anderen drei Gruppen (2,3, 2,1 und 2,6) (Kruskal-Wallis $\mathrm{H}=9,02, \mathrm{df}=3, \mathrm{p}=0,029$ ). In den anderen Prozessfragen ergaben sich aber keine signifikanten Unterschiede.

\section{Diskussion}

In Fortsetzung der Befunde aus der Pilotstudie (Keller et al., 2003) ergab sich, dass die in regelmäßigen Abständen wiederholte Befragung mit einem kurzen Instrument, in dem wichtige Prozessqualitätsaspekte von Zufriedenheit, therapeutischer Zusammenarbeit und Atmosphäre auf einer jugendpsychiatrischen Station abgedeckt werden, neue und aufschlussreiche Ergebnisse gebracht hat.

Die Fragen selbst, auch die neu hinzugefügten zum Bereich Partizipation und Motivation, wurden differenziert beantwortet, wie sich aus den Häufigkeitsangaben der Kategorien ablesen lässt. Eine Hauptkomponentenanalyse erbrachte, dass die Items zu „Mitbestimmung/ernst genommen werden“ wie erwartet einen eigenen Bereich bildeten, der freilich mit der Gesamtzufriedenheit korreliert ist. Der Faktor hat sich als veränderungssensitiv erwiesen, aber nachdem die Befunde mit dem Summenwert nicht wesentlich anders aussahen als bei den Einzelfragen, besonders Frage 3, liegt eine Reduktion der Itemanzahl bei künftigen Verlaufsuntersuchungen nahe. Die Korrelationen zu den ILK-Skalen verweisen auf eine zufrieden stellende externe Validität. Die Fragen wurden auch von den Jugendlichen als adäquat empfunden, und es wurde positiv von ihnen vermerkt, dass sie ernst genommen wurden.

Im Zeitverlauf der Gesamtgruppe ergaben sich nur sehr wenige signifikante Effekte mit Ausnahme bei der Frage nach dem Auskommen mit den Betreuern, das im Laufe des Aufenthaltes abnahm. Hier zeigt sich vermutlich, dass von Seiten der Betreuer nach einer anfänglich eher akzeptierenden Phase zunehmende Anforderungen an die Jugendlichen 
gestellt werden. Bei der Bestimmung der Zufriedenheitstrajektorien während des stationären Verlaufs zeigte sich, dass das Ausgangsniveau bei nahezu allen Fragen sehr unterschiedlich ist. Dies bestätigt die Heterogenität aus der Pilotstudie, während die Befunde zur Veränderung (= Trendkomponente) sehr viel differenzierter als in der Pilotstudie ausfallen. Dort hatten sich kaum unterschiedlich verlaufende Trends gezeigt. Speziell für die generelle Zufriedenheit konnte dies repliziert werden, d.h. die Personen starten auf verschiedenen Niveaus und verbleiben in ihrer Tendenz dort; sie scheinen im „Globalurteil“ somit durch den stationären Aufenthalt wenig beeinflusst zu werden, oder aber sie reagieren im Sinne einer Anpassungsleistung bzw. Übernahme der Patientenrolle, wie es in Zufriedenheitsstudien gemäß Möller-Leimkühler und Dunkel (2003) häufig zu vermuten ist. Die normalerweise damit erklärten hohen Zufriedenheitsbewertungen bei Entlassung sind hier allerdings im Prozesseindruck eher nicht gegeben. Im Bereich der Mitbestimmung und Informiertheit zeigen sich aber sehr wohl zu- und abnehmende Einschätzungen. Die vermutete Stabilität der Zufriedenheitsurteile bestätigt sich daher hauptsächlich im Globalurteil, während Mitbestimmung mit der Zeit zunehmend unterschiedlich erlebt wird. Dabei ist zunehmende Mitbestimmung ja durchaus ein Therapieziel, gerade in dieser Altersgruppe, wo Autonomieentwicklung ansteht. Das Gefühl einer abnehmenden Mitbestimmung wäre daher therapeutisch und motivational sehr ungünstig.

Geschlechtsunterschiede sind insgesamt gering und zeigen sich lediglich in der Tendenz bei der allgemeinen Zufriedenheit und der Mitarbeitsmotivation zu ungunsten der Mädchen. Auch die Korrelationen mit dem Alter sind gering und bestehen v.a. in der Mitbestimmung, wo sich ältere Jugendliche weniger beteiligt sehen. Diagnoseunterschiede hinsichtlich der beiden Hauptgruppen externalisierender und internalisierender Störungen fallen zahlreicher aus. Jugendliche mit externalisierenden Störungen sehen sich als motivierter und kommen insbesondere besser mit den Betreuern und den anderen 
Jugendlichen aus, was einerseits die Wertschätzung der Verlässlichkeit der Betreuer widerspiegeln könnte, aber teilweise auch mit einer mangelnden Wahrnehmung der eigenen sozialen Resonanz erklärbar ist. Generelle Zufriedenheit und Mitbestimmung unterscheiden sich dagegen nicht, so dass diagnostische Unterschiede in dieser Grobeinteilung dennoch wesentlich geringer sind als ursprünglich vermutet. Insgesamt müssen unsere Ergebnisse zu diagnosespezifischen Effekten mit Hinblick auf die beschränkte Stichprobengröße und die deswegen noch sehr heterogenen Diagnosegruppen aber als vorläufig angesehen werden. Auf die Problematik der Konfundierung von Krankheitsaspekten und Selbsteinschätzungen haben im Zusammenhang mit der Lebensqualitätsforschung in der Psychiatrie bereits Mattejat und Remschmidt (1998b) hingewiesen.

Dagegen zeigen sich deutliche Klinikeffekte, zurückzuführen wohl auf die unterschiedliche Behandlungserfahrung in den beiden Teams zum Zeitpunkt der Untersuchung. In beiden Kliniken gelingt es zu Therapiebeginn, eine gute und partnerschaftliche Beziehung aufzubauen, aber dem erfahreneren Team gelingt es dann offenbar besser, gerade bei den internalisierenden Störungen, die Beziehung zu halten und insbesondere das Gefühl der Partizipation wachsen zu lassen. Weitere diagnosespezifische Analysen wären wünschenswert, wurden aber aus Gründen der Stichprobengröße nicht vorgenommen. Als generelle methodische Bemerkung für die Ergebnisinterpretation dieser Studie sei noch erwähnt, dass viele Signifikanztests durchgeführt wurden (alpha-FehlerProblematik), aber auch wegen eventuell zu geringer Teststärke nicht alle Unterschiede zu finden waren (beta-Fehler), so dass nicht jedes Einzelergebnis als gegeben interpretiert werden sollte, dass aber insgesamt die Ergebnisse konsistent und verlässlich scheinen.

Eine weitere Stichprobenvergrößerung, wie sie in folgenden Studien angestrebt wird, dient freilich nicht nur zur Verbesserung der Teststärke, sondern auch zur besseren klinischen Einschätzung von Einzelverläufen. Die individuellen Verläufe können dann vor 
dem Hintergrund ihrer jeweiligen Bezugsgruppe bewertet werden, z.B. hinsichtlich des „Herausfallens“ aus einem Referenzkorridor, der auf den Verläufen einer Vergleichsstichprobe basiert. Die rechtzeitige Entdeckung auffälliger Abweichungen im Therapieverlauf (Lutz et al., 2001) sowie die Entwicklung von Entscheidungsregeln für die Psychotherapie stehen als Ziel hinter diesen Ansätzen, und Lutz et al. (im Druck) beschreiben eine Validierung mittels einer sequenzanalytischen Methode, bei der auch Patientencharakeristika zur Prädiktion herangezogen werden. In der vergleichenden Qualitätssicherung spricht man in diesem Zusammenhang von der Risikoadjustierung, d.h. dem adäquaten Einbeziehen wichtiger Kontextfaktoren zur vergleichenden Bewertung der einzelnen Therapieverläufe. Positiv zu vermerken ist hierzu, dass in unserer Studie grundlegenden Faktoren wie Geschlecht, Alter und Diagnose anscheinend kein allzu großer Einfluss zukommt.

Eine direkte Rückmeldung der Verlaufsergebnisse an die Jugendlichen wurde in Ulm angeboten, aus unterschiedlichen Gründen aber nur unsystematisch und gelegentlich in Anspruch genommen; in Weissenau erfolgte aus technischen Gründen nur sporadisch eine Rückmeldung. Im Wesentlichen wurde daher wohl der „natürliche Verlauf“ erhoben. Eine regelmäßige Zufriedenheitsbefragung in der klinischen Routine als Instrument der Qualitätssicherung im Therapieprozess könnte natürlich andere Daten liefern, und Richter (2004) verweist auf die hohe Bedeutung, wie mit solchen Daten von Seiten der Behandler umgegangen wird und inwieweit eine Rückmeldung der Zufriedenheitsdaten von ihnen als wertvoll für ihre Arbeit eingestuft wird. Bezogen auf diese Studie ging es vorerst darum, die verlaufsrelevanten Items zu identifizieren, sie auf ökonomische Weise abzufragen, um dann zukünftig wenn nötig und möglich im Therapieprozess „gegenzusteuern“.

Die intraindividuelle Variabilität könnte ein weiterer Prädiktor für die Vorhersage des Therapieverlaufs sein. Die gefundene erhöhte Variabilität bei den Jugendlichen mit externalisierenden Störungen lässt sich vermutlich durch ihre Symptomatik erklären, 
indem immer wieder Konflikte entstehen, die Sanktionen nach sich ziehen und somit das Gefühl der Mitbestimmung stark schwanken lassen. Insgesamt ergaben sich aber noch keine klaren Befunde, wohl weil zum einen die zeitliche Auflösung (zweimal wöchentlich) zu grob ist für häufig auftretende Schwankungen, zum anderen weil auch zu wenige Jugendliche mit emotional instabilen Persönlichkeitsstörungen vertreten waren.

Potentiell interessant wäre für zukünftige Studien auch die Korrelation mit dem „outcome“ (z.B. Symptommaßen in Selbst- und Fremdbeurteilung), damit Zufriedenheit nicht nur „Selbstzweck“ ist. Zu erwarten ist freilich eine eher geringe Korrelation bei den Jugendlichen, denn für sie erscheint die Zufriedenheit hauptsächlich durch Erlebensmaße und erst in zweiter Linie durch Erfolgsmaße konstituiert zu sein, während für die Eltern der therapeutische Erfolg im Vordergrund steht (Bredel et al., 2004). Aus Therapeutensicht wiederum sind wahrgenommene Behandlungsmotivation und Kooperation der Jugendlichen hoch mit Verweildauer und Behandlungserfolg korreliert (Schepker et al., 2000). Auch Möller-Leimkühler und Dunkel (2003) verweisen auf die hohe Bedeutung der kommunikativen Qualität der ärztlichen und pflegerischen Betreuung als Indikatoren für die Zufriedenheitseinschätzung erwachsener Patienten, und sie fordern sogar, Leitlinien für die kommunikativen Aspekte der Behandlung zu erarbeiten.

Zusammenfassend unterstreichen die Ergebnisse unserer Studie die Machbarkeit regelmäßiger Patientenbefragungen zur Behandlungszufriedenheit im stationären Therapieprozess. Noch erscheint es verfrüht, eine abschließende Bewertung zur klinischen Relevanz einer solchen Prozessdiagnostik zu treffen. Insbesondere sollten Befragungsintervalle, spezifische Ausrichtung der Fragestellung und Methoden der Integration der Befunde in den Therapieprozess noch weiter erprobt werden. Die globale Zufriedenheit hat sich als insgesamt recht stabil über die gesamte Beobachtungszeit erwiesen, was eine Reduktion der Erhebungszeitpunkte nahe legt. Die Ergebnisse zum Verlauf der von den Jugendlichen wahrgenommenen Partizipation zeigen jedoch, dass es 
sich bei der Behandlungszufriedenheit um ein mehrschichtiges und in Teilen auch dynamisches Konstrukt handelt, das im Sinne der angestrebten Behandlungsmotivation von hoher Bedeutung sein dürfte, so dass sich eine differenzierte regelmäßige Verlaufsbetrachtung auch klinisch lohnen sollte. 


\section{Literatur}

Bredel, S., Brunner, R., Haffner, J. \& Resch, F. (2004). Behandlungserfolg, Behandlungserleben und Behandlungszufriedenheit aus der Sicht von Patienten, Eltern und Therapeuten: Ergebnisse einer evaluativen Studie aus der stationären Kinder- und Jugendpsychiatrie. Praxis der Kinderpsychologie und Kinderpsychiatrie 53, 256-276.

Chatoor, I. \& Krupnick, J. (2001). The role of non-specific factors in treatment outcome of psychotherapy studies. European Child \& Adolescent Psychiatry 10,19-25.

Distler, S. (2002). Behandlungsmotivation, Behandlungszufriedenheit und Lebensqualität aus der Sicht der Eltern an einer kinderpsychiatrischen Einrichtung. Ein Beitrag zur Qualitätssicherung. Praxis der Kinderpsychologie und Kinderpsychiatrie 51, 711-720.

Dür, W., Grossmann, W. \& Schmied, H. (2000). Patientenzufriedenheit und Patientenerwartung im Krankenhaus: Statistische Analysen als Hilfsmittel im Benchmarking. In: M. Bullinger, J. Siegrist \& U. Ravens-Sieberer (Hrsg.), Lebensqualitätsforschung aus medizinpsychologischer und soziologischer Sicht (S. 222-243). Göttingen: Hogrefe.

Freudenmann, R.W. \& Spitzer, M. (2001). Computergestützte Patientenbefragungen als Grundlage für eine moderne Qualitätssicherung in der Psychiatrie: Ergebnisse der Pilotstudien. Nervenarzt 72, 40-51.

Gruyters, T. \& Priebe, S. (1994). Die Bewertung psychiatrischer Behandlung durch die Patienten: Resultate und Probleme der systematischen Erforschung. Psychiatrische Praxis 21, 88-95.

Keller, F. (2003). Analyse von Längsschnittdaten: Auswertungsmöglichkeiten mit hierarchischen linearen Modellen. Zeitschrift für Klinische Psychologie und Psychotherapie 32, 51-61.

Keller, F., Konopka, L., Fegert, J.M. \& Naumann, A. (2003). Prozessaspekte der Zufriedenheit von Jugendlichen in stationär-psychiatrischer Behandlung. Nervenheilkunde 22, 40-46.

Keller, F., Schäfer, S., Konopka, L., Naumann, A. \& Fegert, J.M. (2004). Behandlungszufriedenheit von Kindern in stationär-psychiatrischer Behandlung: Entwicklung und psychometrische Eigenschaften eines Fragebogens. Krankenhauspsychiatrie 15, 3-8.

Konopka, L. (2003). Patienten- und Angehörigenzufriedenheit in der Kinder- und Jugendpsychiatrie: Entwicklung eines Fragebogens. Universität Ulm, unveröff. Dissertation.

Kronmüller, K.-T., Hartmann, M., Reck, C., Victor, D., Horn, H. \& Winkelmann, K. (2003). Die therapeutische Beziehung in der Kinder- und Jugendlichen-Psychotherapie. Zeitschrift für Klinische Psychologie und Psychotherapie 32, 14-23.

Leimkühler, A.M. \& Müller, U. (1996). Patientenzufriedenheit - Artefakt oder soziale Tatsache? Nervenarzt 67, 765-773.

Littell, R.C., Milliken, G.A., Stroup, W.W. \& Wolfinger, R.D. (1996). SAS system for mixed models. Cary, N.C.: SAS Institute Inc.

Lutz, W., Martinovich, Z. \& Howard, K.I. (2001). Vorhersage individueller Therapieverläufe. Zeitschrift für Klinische Psychologie und Psychotherapie 30, 104-113.

Lutz W., Tholen, S., Kosfelder, J., Grawe, K. \& Schulte, D. (im Druck). Zur Entwicklung von Entscheidungsregeln in der Psychotherapie: Die Validierung von Vorhersagemodellen mit einer sequenzanalytischen Methode. Zeitschrift für Klinische Psychologie und Psychotherapie.

Mattejat, F., Jungmann, J., Meusers, M., Moik, C., Nölkel, P., Schaff, C., Scholz, M., Schmidt, M.H. \& Remschmidt, H. (1998). Das Inventar zur Erfassung der Lebensqualität bei Kindern und Jugendlichen (ILK) - Eine Pilotstudie. Zeitschrift für Kinder- und Jugendpsychiatrie 26, 174-182.

Mattejat, F. \& Remschmidt, H. (1998a). Fragebögen zur Beurteilung der Behandlung (FBB). Göttingen: Hogrefe. 
Mattejat, F. \& Remschmidt, H. (1998b). Zur Erfassung der Lebensqualität bei psychisch gestörten Kindern und Jugendlichen - eine Übersicht. Zeitschrift für Kinder- und Jugendpsychiatrie 26, 183196.

Möller-Leimkühler, A.M. \& Dunkel, R. (2003). Zufriedenheit psychiatrischer Patienten mit ihrem stationären Aufenthalt. Nervenarzt 74, 40-47.

Raudenbush, S.W. (2001). Comparing personal trajectories and drawing causal inferences from longitudinal data. Annual Review of Psychology 52, 501-525.

Richter, D. (2004). Qualitätsindikatoren für die psychiatrische Versorgung: Eine Übersicht über Kriterien, Methoden und Probleme. Krankenhauspsychiatrie 15, 104-113.

Schepker, R., Wirtz, M. \& Jahn, K. (2000). Verlaufsprädiktoren mittelfristiger Behandlungen in der stationären Kinder- und Jugendpsychiatrie. Praxis der Kinderpsychologie und Kinderpsychiatrie 49, 656-676.

Singer, J.D. (2002). Fitting individual growth models using SAS Proc Mixed. In: D.S. Moskowitz \& S.L. Hershberger (Eds.), Modeling intraindividual variability with repeated measures data: Methods and applications (S. 135-170). Mahwah, NJ: Lawrence Erlbaum Ass.

Teague, G. (2000). Patient perceptions of care measures. In: American Psychiatric Association (Ed). Handbook of psychiatric measures (pp 169-176). Washington DC: APA.

Verbeke, G. \& Molenberghs, M. (2000). Linear mixed models for longitudinal data ( $2^{\text {nd }}$ ed.). New York: Springer.

Weisz, J.R. \& Jensen, A.L. (2001). Child and adolescent psychotherapy in research and practice contexts: Review of the evidence and suggestions for improving the field. European Child \& Adolescent Psychiatry 10, 12-18.

Weisz, J.R. \& Kazdin, A.E. (2003). Present and Future of Evidence-Based Psychotherapies for Children and Adolescents. In: A.E. Kazdin \& J.R. Weisz (Eds.) Evidence-Based Psychotherapies for Children and Adolescents (pp 439-451). New York: Guilford.

Wiethoff, K., Dippold, I., Rothärmel, S., Wolfslast, G., Konopka, L., Naumann, A., Keller, F. \& Fegert J.M. (2003). "Ich durfte ja nichts sagen, ich musste hier rein" - Bedingungen und Folgen der stationären Aufnahme aus der Sicht minderjähriger Patienten. In: U. Lehmkuhl (Hrsg.), Ethische Grundlagen in der Kinder- und Jugendpsychiatrie und Psychotherapie (S. 89-104). Göttingen: Vandenhoeck \& Ruprecht. 
Tabelle 1: Mittelwerte und Standardabweichungen (SD) zu den Einzelfragen über alle Zeitpunkte ( $t=783 / 360 / 391)$ und Ergebnisse einer Hauptkomponentenanalyse ${ }^{\mathrm{a}}$ (varimaxrotiert) der Daten zum jeweils dritten Zeitpunkt $(n=73)$

\begin{tabular}{lccccc}
\hline \multicolumn{1}{c}{ Frage (Kurzform) } & $\begin{array}{c}\text { Mittel- } \\
\text { wert }\end{array}$ & SD & Faktor 1 & Faktor 2 & Faktor 3 \\
\hline 1) insgesamt ging es mir gut & 3,60 & $(1,3)$ & .42 & .77 & \\
2) insgesamt zufrieden & 3,39 & $(1,4)$ & .31 & .86 & \\
3) Dinge mitbestimmen & 3,38 & $(1,3)$ & & .46 & .65 \\
4) mit Therapien zufrieden & 4,06 & $(1,1)$ & .82 & & \\
5) mit Betreuern ausgekommen & 4,04 & $(1,1)$ & .47 & .53 & .41 \\
6) mit Jugendlichen verstanden & 4,27 & $(0,9)$ & .43 & .55 & \\
7) von Betreuern ernst genommen & 3,68 & $(1,2)$ & & & .83 \\
8) von Therapeuten ernst genommen & 4,10 & $(1,1)$ & & & .83 \\
9) über Behandlung informiert & 3,46 & $(1,4)$ & .56 & & \\
10) sinnvoll, dass weiter hier & 3,74 & $(1,4)$ & .55 & & \\
11) Lust, mitzuarbeiten bei Therapien & 3,76 & $(1,3)$ & .93 & & - \\
12) Medikamente helfen & 3,46 & $(1,3)$ & - & - & - \\
13) Nebenwirkungen gespürt & 1,95 & $(1,3)$ & - & - & \\
14) Problemen ausgeliefert & 3,26 & $(1,4)$ & .48 & -.62 & \\
15) Essen geschmeckt & 3,22 & $(1,5)$ & & .41 & \\
16) Privatsphäre respektiert & 3,66 & $(1,3)$ & & & .61 \\
\hline
\end{tabular}

a) dargestellt sind nur |Ladungen| > .30; die Medikamentenfragen wurden nicht aufgenommen, da sie nur für eine Subgruppe relevant sind. 
Tabelle 2: Zusammenhänge ${ }^{a}$ zwischen den Prozessfragen und einzelnen Dimensionen ${ }^{b}$ des Inventars zur Lebensqualität (ILK-J, Selbsteinschätzung) für die ersten 60 Tage $(\mathrm{t}=378)$

\begin{tabular}{|c|c|c|c|c|c|c|c|}
\hline \multirow[b]{2}{*}{ Prozessfragen } & \multicolumn{7}{|c|}{ ILK - Dimension ${ }^{\mathrm{b}}$} \\
\hline & ILK 3 & ILK 4 & ILK 5 & ILK 6 & ILK 7 & ILK 8 & ILK 9 \\
\hline 1) insgesamt ging gut & & &,- 25 &,- 42 &,- 55 &,- 32 &,- 34 \\
\hline 2) insgesamt zufrieden & & &,- 32 &,- 52 &,- 67 &,- 40 &,- 31 \\
\hline 3) Dinge mitbestimmen &,- 20 & &,- 19 &,- 24 &,- 33 & &,- 21 \\
\hline 4) mit Therapien zufrieden & &,- 16 & &,- 26 &,- 35 &,- 20 &,- 29 \\
\hline 5) mit Betreuern ausgekommen &,- 27 & &,- 22 &,- 28 &,- 39 &,- 20 &,- 32 \\
\hline 6) mit Jugendlichen verstanden &,- 38 & &,- 15 & &,- 21 & & \\
\hline 7) von Betreuern ernst genommen &,- 19 & &,- 16 &,- 23 &,- 25 &,- 17 &,- 30 \\
\hline 8) von Therap. ernst genommen &,- 25 &,- 22 &,- 15 &,- 23 &,- 22 & &,- 25 \\
\hline 9) über Behandlung informiert &,- 15 & &,- 17 &,- 24 &,- 32 & &,- 26 \\
\hline 10) sinnvoll, dass weiter hier & & & & & & ,16 &,- 23 \\
\hline 11) Lust, mitzuarbeiten bei Ther. &,- 19 & &,- 21 &,- 35 &,- 44 &,- 26 &,- 27 \\
\hline 12) Medikamente helfen & & &,- 19 &,- 38 &,- 40 &,- 26 &,- 23 \\
\hline 13) Nebenwirkungen gespürt & & & & & & &,- 16 \\
\hline 14) Problemen ausgeliefert & & ,19 & ,31 &, 52 &, 49 &, 54 & \\
\hline 15) Essen geschmeckt & & &,- 24 &,- 15 &,- 31 & &,- 23 \\
\hline 16) Privatsphäre respektiert &,- 19 & &,- 21 &,- 23 &,- 28 & &,- 25 \\
\hline
\end{tabular}

a) Spearman-Rangkorrelationen; dargestellt sind nur $|r|>.15$ (fett gedruckt, wenn $>$.30)

b) Bezeichnung der ILK-Dimensionen:

ILK-3: Soziale Kontakte mit Gleichaltrigen

ILK-4: Interessen und Freizeitgestaltung

ILK-5: Körperliche Gesundheit

ILK-6: Psychische Gesundheit

ILK-7: Gesamtbeurteilung der Lebensqualität

ILK-8: Belastung durch die aktuelle Krankheit

ILK-9: Belastung durch die diagnostischen und therapeutischen Maßnahmen 
Tabelle 3: Ergebnisse zum Gesamtverlauf und bezüglich der Gruppenunterschiede nach Klinik und diagnostischer Unterscheidung sowie die jeweiligen Interaktionseffekte Gruppe $x$ Zeit $(p$-Werte <.10).

\begin{tabular}{|c|c|c|c|c|c|c|}
\hline \multirow[b]{2}{*}{ Inhalt der Fragen } & \multirow[b]{2}{*}{$\begin{array}{l}\text { Homogenität } \\
\text { der Steigungen }\end{array}$} & \multirow[b]{2}{*}{$\begin{array}{l}\text { Haupteffekt } \\
\text { Zeit }\end{array}$} & \multicolumn{2}{|c|}{ Klinik } & \multicolumn{2}{|c|}{ Diagnose } \\
\hline & & & $\begin{array}{c}\text { Ulm I } \\
\text { Weissenau }\end{array}$ & $\begin{array}{c}\text { Interaktion } \\
\text { mit Zeit }\end{array}$ & $\begin{array}{l}\text { internal./ } \\
\text { external. }\end{array}$ & $\begin{array}{c}\text { Interaktior } \\
\text { mit Zeit }\end{array}$ \\
\hline 1) insgesamt ging gut & .053 & & & & & \\
\hline \multicolumn{7}{|l|}{ 2) insgesamt zufrieden } \\
\hline 3) Dinge mitbestimmen & .049 & .059 & & .0005 & & \\
\hline 4) mit Therapien zufrieden & & & & & .016 & \\
\hline 5) mit Betreuern ausgekommen & .022 & $<.001$ & & .026 & .036 & \\
\hline 6) mit Jugendlichen verstanden & & & & .088 & .012 & \\
\hline 7) von Betreuern ernst genommen & .002 & .078 & & & & \\
\hline 8) von Therapeuten ernst genommen & .011 & & & & & \\
\hline 9) über Behandlung informiert & .006 & & & .027 & & .049 \\
\hline 10) sinnvoll, dass weiter hier & $<.001$ & & & & .019 & .016 \\
\hline 11) Lust, mitzuarbeiten bei Therapien & & & & & .007 & \\
\hline 12) Medikamente helfen & .030 & .062 & & & & .002 \\
\hline 13) Nebenwirkungen gespürt & .091 & & & & & \\
\hline 14) Problemen ausgeliefert & .003 & & & & .10 & \\
\hline 15) Essen geschmeckt & .017 & & & .059 & & \\
\hline 16) Privatsphäre respektiert & .001 & & & .097 & & .046 \\
\hline
\end{tabular}


Korrespondenzadresse:

PD Dr. Ferdinand Keller, Dipl.-Psych.

Klinik für Kinder- und Jugendpsychiatrie/Psychotherapie

Universitätsklinikum Ulm

Steinhövelstr. 5

89075 Ulm

Tel.: 0731 / 500-33588

email: Ferdinand.Keller@uniklinik-ulm.de

Danksagung:

Wir danken Martin Chmelnik für seine Mithilfe bei der Datenerhebung und Jörg Sigle für die technische Beratung beim Einsatz seines computergestützten Befragungsprogramms AnyQuin.

Die Laptops für die Befragung wurden im Rahmen einer Sachmittelspende von Janssen-Cilag zur Verfügung gestellt. 